\title{
Los entornos activo-colaborativos de aprendizaje como buenas prácticas en el desarrollo de competencias transversales en la formación profesional de la Comunidad Autónoma del País Vasco
}

\author{
Ana Eizagirre Sagardia \\ Jon Altuna Urdin \\ Idoia Fernández Fernández \\ Euskal Herriko Unibertsitatea / Universidad del País Vasco. España. \\ a.eizagirre@ehu.eus \\ jon.altuna@ehu.eus \\ idoia.fernandez@ehu.eus
}

Recibido: 26/2/2016

Aceptado: 17/4/2016

Publicado: 26/10/2017

\begin{abstract}
Resumen
Este artículo analiza el modo en que las orientaciones prescriptivas generales sobre competencias transversales se están llevando realmente a la práctica en los centros de formación profesional de la Comunidad Autónoma Vasca. Se realiza un estudio de caso en el que se transita desde una detección previa de carácter cuantitativo hasta un análisis cualitativo de las prácticas en las que las citadas competencias han sido desarrolladas con éxito según la percepción del profesorado, del alumnado y de los equipos directivos implicados. Como resultado, se han identificado tres niveles de sistematización y desarrollo institucional de las competencias transversales: el individual, el de ciclo y el de centro. La comparación entre estas tipologías resultantes revela que las prácticas innovadoras a nivel de ciclo, y más en concreto en los ciclos de alto rendimiento (ETHAZI), concentran unas características que representan un salto cualitativo en la concepción, el desarrollo metodológico y la evaluación de las citadas competencias, y que, por tanto, pueden servir de guía para ir superando los obstáculos que, a nivel práctico, encuentra el desarrollo real de las competencias transversales en el ámbito de la formación profesional.
\end{abstract}

Palabras clave: aprendizaje colaborativo; desarrollo de competencias; innovación educativa; educación secundaria; educación superior

Resum. Els entorns activocol-laboratius de l'aprenentatge com a bones pràctiques en el desenvolupament de competències transversals dins la formació professional de la Comunitat Autònoma Basca

Aquest article analitza la manera com les orientacions prescriptives generals sobre competències transversals s'estan portant realment a la practica als centres de formació professional de la Comunitat Autònoma Basca. S’hi realitza un estudi de cas en el qual es transita des d'una detecció prèvia de caràcter quantitatiu fins a una anàlisi qualitativa de les pràc- 
tiques en les quals les esmentades competències han estat desenvolupades amb èxit segons les percepcions del professorat, de l'alumnat i dels equips directius implicats. Com a resultat, s'han identificat tres nivells de sistematització i desenvolupament institucional de les competències transversals: l'individual, el de cicle i el de centre. La comparació entre aquestes tipologies resultants posen de relleu que les pràctiques innovadores a nivell de cicle, i mes en concret en els cicles d'alt rendiment (ETHASI), concentren unes característiques que representen un salt qualitatiu en la concepció, el desenvolupament metodològic i l'avaluació de les esmentades competències, i que, per tant, poden servir de guia per anar superant els obstacles que, a nivell pràctic, troba el desenvolupament real de les competències transversals en l'àmbit de la formació professional.

Paraules clau: aprenentatge col-laboratiu; desenvolupament de competències; ensenyament secundari; innovació educativa; ensenyament superior

\section{Abstract. Active-collaborative learning as best practices in the development of cross-curricular competencies in Basque Country vocational training}

This article analyzes how the general prescriptive guidelines on cross-curricular competencies are being implemented in vocational training centers of the Basque Autonomous Community. A case study is carried out by means of a quantitative and qualitative analysis of good practices in which competencies have been successfully developed according to the perceptions of the teachers, students and management teams involved. Systematization and institutional development of cross-curricular competences were identified at three levels: the individual level, the cycle level and the center level. The comparison between these typologies reveals that innovative practices at the cycle level, specifically in high performance cycles (the ETHAZI model), have resulted in a qualitative leap in the design, methodological development and assessment of these competencies and can serve at the practical level as a guide to overcome obstacles in the actual development of cross-curricular competencies in vocational training.

Keywords: collaborative learning; competencies development; educational innovation; secondary education; higher education

\section{Sumario}
1. Introducción
4. Discusión y conclusiones
2. Metodología
Referencias bibliográficas

3. Resultados

\section{Introducción}

La reflexión acerca de los niveles suficientes de capacitación que posibiliten a las personas realizar una socialización adecuada en un contexto de cambio acelerado y global ha generado, en las últimas décadas, el surgimiento del concepto de competencia y el establecimiento de una serie de competencias clave, así como de competencias transversales o genéricas (Perrenoud, 2004; Gimeno Sacristán, 2008; Monereo, 2009; Poblete, Bezanilla, Fernández-Nogueira y Campo, 2016; Villardón-Gallego, 2015), convertidas paulatinamente en el eje orientador de la práctica pedagógica en los diversos niveles de enseñanza (Bolí- 
var, 2008) y de las políticas generales de educación de la Unión Europea (Proyecto Tuning, 2003; OCDE, 2005; European Commission, 2012).

En líneas generales, las competencias transversales (CT) suponen la integración de conocimientos, habilidades, actitudes y valores (Ott, 1999) que una persona pone en juego en una situación concreta, a fin de demostrar que es capaz de resolverla. A pesar de lo borroso que resulta a veces su conceptualización, la competencia se afianza cada vez más como un concepto útil para profundizar en el espacio que hay entre la formación académica y los requerimientos formativos reclamados en el mercado de trabajo (Prahalad y Hamel, 1990; Mulder, 2007; Rué, 2009), así como la piedra fundamental sobre la que ha de construirse esa nueva educación y formación para que se haga posible la transformación tan reclamada en esta sociedad del conocimiento y de cambio globalizado (Hargreaves, 2003).

En el número monográfico que la Revista Europea de Formación Profesional dedicaba a la formación profesional basada en competencias, Grootings (1994) concluía que el paradigma basado en competencias había surgido de las nuevas maneras de organizar el trabajo y como consecuencia de las nuevas maneras de seleccionar a los empleados, lo que suponía la necesidad de integrar este enfoque en los programas de formación profesional. En su análisis, Arguelles y Gonczi (2000) constataban, en la misma línea, que la educación basada en competencias se estaba convirtiendo, cada vez en más países, en el modelo a seguir para solucionar los problemas de la formación profesional. La formación profesional basada en competencias se convertía también en uno de los ejes de trabajo centrales del CEDEFOP (Descy y Tessaring, 2001). A partir del año 2000, el Education and Competence Studies Group, de la Universidad de Wageningen (Holanda), destaca en su análisis comparado de países como Reino Unido, Alemania, Francia y Países Bajos, que, con diferentes enfoques y énfasis, el sistema se ha extendido y se ha convertido en el eje de las políticas de FP.

Las competencias están relacionadas con las actitudes profesionales, la reflexividad sobre el propio comportamiento, el desarrollo de la carrera laboral, la relación entre el mundo de la educación y el trabajo, el reconocimiento de competencias adquiridas informalmente, la autonomía y la capacidad de desenvolverse en un mundo de cambios constantes y profundos. Son, en parte, estas competencias las que condicionan el nivel de empleabilidad de una persona (Rodríguez, 2012). Por ello, la FP debe asumir la responsabilidad de capacitar a los y las estudiantes para que puedan desarrollar efectivamente una actividad profesional y adquieran las competencias necesarias que faciliten su empleabilidad (Bridge, O'Neill y Cromie, 2003; Knight y Yorke, 2003).

Esta nueva orientación llegó también a España en 1999, cuando se creó el Instituto Nacional de las Cualificaciones (INCUAL). La Ley Orgánica 5/2002 de las cualificaciones y de la formación profesional, posteriormente modificada por la Ley 2/2011 de economía sostenible, son los marcos normativos que intentan desarrollar la formación reglada, ocupacional y continua, así como trazar itinerarios laborales para los trabajadores, lo que permite un reconoci- 
miento de competencias adquiridas y facilita la movilidad en la formación y en el empleo.

En el País Vasco, la FP basada en competencias ha ido creciendo, favorecida por las políticas desplegadas a través del Instituto Vasco de las Cualificaciones y Formación Profesional (Decreto 119/1998, de 23 de junio) y de la Agencia Vasca para la Evaluación de la Competencia y de la Calidad de la Formación Profesional (Decreto 62/2001, de 3 de abril). En 2005, se puso en marcha el Centro de Innovación para la Formación Profesional y el Aprendizaje Permanente (TKNIKA), que desempeña un papel fundamental en la dinamización de la innovación metodológica, poniendo especial atención en el desarrollo de las competencias transversales. Su aportación se une así a la realizada por la Asociación de Centros de Formación Profesional (HETEL, 2008), que puso de relieve diez competencias básicas en el desarrollo de los ciclos formativos, dando orientaciones para su desarrollo metodológico y su evaluación. La Confederación Empresarial Vasca (Confebask, 2010) destacó también trece competencias en las personas trabajadoras vascas que reforzaban esta misma idea, con lo que hacía suya la importancia de involucrar a los agentes económicos en la construcción de este proceso (Weigel, Mulder y Collins, 2007a).

El discurso basado en competencias supone, por otra parte, un cuestionamiento de los contenidos del currículo y del proceso de enseñanza-aprendizaje, y resalta la necesidad de realizar innovaciones pedagógicas que lo faciliten (European Commission/EACEA/Eurydice, 2012). Cobran especial importancia las tareas de aprendizaje porque deben integrar conocimientos, destrezas y actitudes, además de estar dotadas de un carácter de autenticidad, es decir, ser relevantes, reflejar la complejidad de la realidad y anclar con tareas de mayor envergadura. Este cambio en la arquitectura docente conduce, asimismo, a prestar atención a la evaluación de los resultados de aprendizaje y a la calificación de los productos derivados de las tareas auténticas (Gulikers, 2006; Blanco, 2008).

En este contexto, surgen nuevas propuestas metodológicas (activas), a menudo interdisciplinares, donde el alumnado es el centro del proceso formativo, con lo que se supera así el sistema tradicional de enseñanza basado en la docencia de las disciplinas (Imbernon y Medina, 2006; Moya, 2008). El aprendizaje basado en proyectos, el estudio de caso, el aprendizaje colaborativo, etc. son algunas de las metodologías que parecen adecuadas para este enfoque, que están siendo utilizadas en cada vez más estudios universitarios (De Miguel, 2005; Benito y Cruz, 2005; Fernández, 2006), en formación profesional (Rauner, 2007) y en la investigación pedagógica (Arias-Gundin, Fidalgo y García, 2008).

Diseñar e implementar una FP basada en competencias se presenta, por lo tanto, como una tarea compleja que, según el anteriormente citado Education and Competences Studies Group, supone superar una serie de escollos tales como: la diversidad en la percepción sobre el concepto de competencia, la excesiva tendencia a la estandarización, la relación entre la escuela y la empresa, el diseño y la implementación de corte tradicional, la evaluación, el nuevo 
rol del profesorado y el rediseño de la organización y la gestión de los centros (Biemans, Wesselink, Gulikers, Schaafsma, Verstegen y Mulder, 2009).

La conclusión es que el modelo basado en competencias se ha convertido en una tendencia, pero todavía no hay suficientes líneas de orientación, instrucciones ni ejemplos que ayuden a comprender las claves que subyacen en las prácticas más efectivas y exitosas. Los escollos que presenta la materialización práctica de un currículo basado en competencias son la punta de un iceberg que necesita ser investigado en profundidad, de manera que las políticas, los programas y las prácticas se sustenten sobre bases más sólidas y científicas.

¿Qué está ocurriendo en los centros de FP en lo que respecta a las competencias transversales? ¿Hasta qué punto el profesorado y los centros vascos están materializando prácticas en las que las competencias transversales se desarrollan de manera adecuada? ¿Cómo son estas prácticas y qué lecciones se pueden extraer de ellas? Con estos interrogantes, se puso en marcha el proyecto de investigación Gaitasunez, que pretendía analizar y comprender en profundidad el modo en que las orientaciones prescriptivas generales sobre competencias transversales procedentes de marcos internacionales, estatales y/o autonómicos se estaban llevando realmente a la práctica en los centros de formación profesional de la Comunidad Autónoma Vasca.

Este proyecto se centró en el análisis detallado de las buenas prácticas detectadas dentro del estudio de caso de la FP vasca, desde una perspectiva abierta, conscientes del carácter complejo y multidimensional de la propia expresión de «buenas prácticas» (Escudero, 2009), y considerando que las mismas están llamadas a ser interpretadas y reconstruidas por los individuos en sus contextos y en sus prácticas, más que a ser aplicadas linealmente y menos impuestas por decreto. Esta perspectiva permitió operar dentro de la diversidad del contexto y distinguir, como veremos más adelante, tres casos tipo (desarrollados a nivel individual del profesorado, a nivel de ciclo y a nivel general de centro) que ofrecen un modelo de buenas prácticas para ayudar a los docentes a situar su realidad, a reflexionar y a proponer procesos de cambio y mejora.

En este artículo, nos centraremos en el caso tipo que opera desde el ciclo, en concreto, la experiencia desarrollada dentro del programa de formación denominado ciclos de alto rendimiento (Etekin Handiko Zikloak ETHAZI ${ }^{1}$ ), que pretende formar e impulsar, entre el profesorado, el trabajo en entornos activo-colaborativos de aprendizaje basado en retos. El caso tipo de ciclo sirve de ejemplo para comprender cómo, partiendo de realidades típicas basadas en el aprendizaje disciplinario técnico, se construyen formas alternativas de currículo donde las competencias transversales adquieren un significado más global e integrado.

1. Tras un primer periodo de experiencia piloto durante el curso 2013-2014, donde participaron 5 ciclos formativos de otros tantos centros de FP, son 25 los nuevos centros que han dado los primeros pasos para implementar dicho modelo en sus aulas. En la actualidad, el programa ETHAZI es uno de los pilares sobre los que se asienta la línea de actuación en materia de innovación pedagógica recogida en el IV Plan Vasco de Formación Profesional aprobado por el Gobierno Vasco en diciembre de 2014 e impulsado por TKNIKA. 


\section{Metodología}

El punto de partida fue centrar la atención en toda la red de centros de FP de la Comunidad Autónoma Vasca y aplicar un método que permitiera realizar un análisis pormenorizado de sus centros. Se optó por llevar a cabo un estudio de caso que permitió explorar el fenómeno en profundidad dentro de sus escenarios naturales (Bogdan y Biklen, 1982; Stake, 1998; Vázquez y Angulo, 2003), así como comprender en detalle las diferentes realidades educativas (Yin, 2003) y sus particularidades. El caso lo constituyen, por tanto, todos los procesos particulares de desarrollo de competencias transversales que se articulan en los centros de FP objeto de investigación.

La aproximación al contexto y a las prácticas de éxito se llevó a cabo, en primera instancia, a través de técnicas cuantitativas, con el objetivo de realizar un mapa de situación que ayudara a identificar los centros de formación profesional de la Comunidad Autónoma Vasca que trabajan las competencias transversales de forma sistemática. A partir de esta primera aproximación, se trabajó con técnicas cualitativas, escuchando de cerca las voces de los protagonistas de este fenómeno (profesorado y alumnado) y captando los matices que nos ayudaron tanto en la detección como en la caracterización e interpretación de las prácticas de éxito.

\subsection{Sujetos participantes de la investigación}

La selección de centros y de participantes de la investigación fue variando en función de las fases del proyecto. En la primera parte de identificación de centros, participaron 473 docentes (de una población finita de 5.500) de 48 de los 61 centros de FP, que contestaron a un cuestionario en línea sobre las competencias transversales trabajadas en sus centros educativos. Aunque el número de respuestas por centro era bastante heterogéneo, estas fueron contrastadas con el personal técnico de TKNIKA y sirvieron para realizar un primer filtro de aquellos en los que se trabajan las competencias transversales de manera más o menos sistemática.

En la segunda fase, participaron 177 estudiantes, 16 equipos directivos y 21 docentes (pertenecientes a 7 centros) mediante grupos de discusión, entrevistas grupales y relatos comunicativos, técnicas que permitieron registrar sus experiencias y sus opiniones sobre quiénes, y cómo desarrollan las CT a nivel de centro, de familia profesional, de ciclo y de asignatura.

En el caso de las experiencias ETHAZI, participó el 100\% del alumnado y del profesorado de dos ciclos, así como una representación variable del alumnado de los otros tres ciclos implicados en el curso 2013-2014.

\subsection{Fases del proyecto y técnicas de recogida y de análisis de datos}

Las fases de la investigación surgieron de la necesidad de identificar las prácticas de éxito y de ubicar aquellas que fueron consideradas como tales. Se redujo la población inicial (todo el profesorado de formación profesional de 
la Comunidad Autónoma Vasca) a un número mucho menor de enseñantes que, según las fuentes de contraste (equipos directivos y alumnado), fueron considerados sujetos de prácticas de éxito.

La primera fase conjugó la revisión teórica con la investigación empírica a través de un cuestionario en línea (Google.docs) dirigido a todo el profesorado de FP de la Comunidad Autónoma Vasca, de manera que pudimos conocer en qué centros se había producido un desarrollo sistemático de las CT.

La segunda fase profundizó en la identificación y en la caracterización de las prácticas de éxito utilizando técnicas cualitativas. En concreto:

- Entrevistas grupales a los equipos directivos de los centros objeto de estudio (Bisquerra, 2004), para contrastar resultados del cuestionario y conocer el planeamiento institucional que cada centro tenía para las CT.

- Grupos de discusión con el profesorado de aquellos centros en los que el trabajo en competencias transversales cuenta con un marco de apoyo institucional y pedagógico, bien a nivel de ciclo o de centro. Se generó un discurso grupal en el que identificar, contrastar y debatir distintas tendencias y regularidades en sus opiniones sobre el desarrollo de las CT (Bisquerra, 2004; Krueger, 1991).

- Entrevistas en profundidad al profesorado cuyas prácticas fueron consideradas de éxito (por parte de ellos y de ellas mismos, del equipo directivo y del alumnado), pero que, sin embargo, no contaban con un marco institucional más global. Fueron narraciones reflexionadas que sirvieron tanto para detectar aspectos del presente y del pasado como expectativas de futuro respecto al objeto de estudio (Gómez, Latorre, Sánchez y Flecha, 2007).

Entre ambas fases, se llevaron a cabo los grupos de discusión con el alumnado, con objeto de validar los datos derivados del cuestionario (y provocar el descarte de los centros en los que las percepciones de profesorado y alumnado no fueron coincidentes) y validar e interpretar igualmente los datos cualitativos producidos en la caracterización de las prácticas de éxito.

Se establecieron unas dimensiones generales que guiaron las entrevistas y los grupos de discusión, además, se preparó un guión semiestructurado específico para cada colectivo, donde se recogen cuestiones relacionadas con las formas de percibir y definir las competencias transversales, las formas de concebir la enseñanza, las relaciones con el alumnado, las dificultades a la hora de trabajar las CT, las claves y los factores de éxito, etc. Para codificar y analizar los datos, se utilizó el programa NVivo (versión 10), que facilitó el manejo y la organización de datos cualitativos (Sánchez Gómez y García-Valcárcel Muñoz-Repiso, 2001), así como la construcción del sistema categorial. Se realizaron matrices de intersección de cada una de las dimensiones en base a los atributos de los participantes.

Se trabajó con criterios de credibilidad, transferibilidad, confirmabilidad y ética (Guba y Lincoln, 1985: 85). Los aspectos éticos se gestionaron a través de un protocolo de consentimiento informado con todas las personas partici- 
pantes, en el que se explicaba la finalidad investigadora y su tratamiento posterior de acuerdo con la Ley de protección de datos de carácter personal del 13 de diciembre de 1999. En todo el proceso de investigación, se mantuvo el anonimato de los participantes.

\section{Resultados}

El análisis de los datos generales muestra una gran diversidad de prácticas de éxito, considerando como tales aquellas en las que convergen las opiniones, las percepciones y/o las valoraciones positivas de alumnado, profesorado y equipos directivos. Ahora bien, dentro de esta diversidad, se identificaron tres niveles de sistematización y desarrollo institucional de las CT que permiten perfilar estos tres casos tipo:

I. Caso tipo 1. Desarrollo de competencias transversales a nivel de profesorado individual: casos en los que el alumnado reconoce un trabajo de CT en algún módulo o con algún profesor o profesora en concreto. Se producen de forma más o menos aislada y difusa, ya que no hay una política explícita de centro encaminada a desarrollarlas. La mayoría de los centros entrevistados responden a esta tipología.

II. Caso tipo 2. Desarrollo de competencias transversales a nivel de ciclo, en las que todo el profesorado de un ciclo está inmerso en dicha actividad. La inmersión se da en un entorno de cambio metodológico más general de aprendizaje basado en proyectos o retos. En la mayoría de estos casos, el trabajo de ciclo está vinculado actualmente al programa de ciclos de alto rendimiento dirigido por TKNIKA. Una cuarta parte de los centros entrevistados respondería a esta tipología.

III. Caso tipo 3. Desarrollo de competencias transversales a nivel de centro, en el que hay explicitado cierto grado de sistematización de las CT a aplicar en toda la institución educativa. Los casos tipo 3 (dos centros) se caracterizan por tener una política propia en relación con el desarrollo de las CT, de manera que se ha convertido en una característica institucional suya. En estos centros, conviven ciclos de alto rendimiento junto con ciclos que mantienen una estructura organizativa más tradicional.

En el presente artículo, vamos a centrarnos en aquellos aspectos que caracterizan específicamente a los casos tipo 2, tipología de ciclo, y, en concreto, en las experiencias de los ciclos de alto rendimiento, analizando principalmente aquellas características relacionadas con la conceptualización y la sistematización y el proceso de enseñanza-aprendizaje.

\subsection{Conceptualización de las competencias transversales}

En los ciclos de alto rendimiento, el equipo de docentes goza de una gran autonomía y autodeterminación y decide las competencias que considera 
fundamentales para el alumnado. Es difícil establecer cómo se produce el tránsito de formas de trabajo individuales a otras más colegiadas. Muy probablemente, no hay un solo patrón, pero, en el plano más operativo, el profesorado de ciclo comienza a trabajar las $\mathrm{CT}$ en base a criterios particulares, como, por ejemplo, que sean fáciles de puntuar en el día a día y que se valoren en todos los módulos.

Se observa que, en los momentos iniciales, las competencias transversales se asocian repetidamente a las actitudes, y la preocupación de los docentes es cómo evaluarlas y calificarlas manteniendo unos criterios comunes coherentes y factibles. Se trata de acercamientos analíticos y predeterminados, es decir, se adoptan una serie de actitudes (o CT) como importantes y se trata de buscar la manera que facilite que todo el equipo docente pueda controlarlas una a una.

Eso fue desde el principio. Veíamos que la actitud del alumnado debía de ser la misma en todos los módulos y entonces cogimos todas las actitudes y empezamos a filtrarlas, filtrarlas y filtrarlas, las que podían ser fácilmente puntuables en el día a día y las que podían ser valoradas en todos los módulos, e hicimos una tabla de actitudes transversales a todos los módulos, y a esta le dimos el 30\%. (Profesor 19, T2)

En otros casos, el criterio que guía la selección de las competencias transversales será la estructura que debería tener el recorrido del profesional en cuestión. Se trata de un acercamiento más sofisticado que fija la atención en el perfil de salida y lo que supuestamente es necesario en el mundo laboral, y que busca una lógica curricular que dé sentido a ese itinerario. Estamos, sin duda, en una orientación educativa más conectada con el concepto de empleabilidad. Es una proyección más gráfica e integrada de búsqueda de sentido.

Es una rueda. Se trata de expresar, en un pictograma o en una imagen, dónde están las competencias de un alumno o de una alumna y cómo deberían entrenarse [...]. Sí. No las competencias más concretas, pero sí qué estructura debería tener el itinerario de un alumno, de un trabajador o de una persona. (Profesor 13, caso tipo 2)

Se observa, por tanto, que el trabajo a nivel de ciclo desencadena procesos reflexivos en el equipo docente, que se va alejando de los modos más mecánicos de tratar las competencias transversales, a la vez que gana en autonomía y en capacidad de autogestión pedagógica. Uno de los resultados más relevantes es la importancia que otorga el profesorado a interpretar el significado de las CT en cada familia o ciclo, es decir, los ciclos de alto rendimiento han realizado una lectura contextualizada de sus CT adaptándolas a su cultura profesional y a las necesidades reales del entorno en que se encuentran.

La manera de pensar sobre las competencias transversales que manifiesta este profesorado es mucho más rica, contextual y plástica que la que habitualmente se marca en los documentos prescriptivos (autonómicos, estatales o europeos). En base a criterios como las necesidades del perfil profesional, la tipología del alumnado (ciclo medio o ciclo superior), la tradición y la cultu- 
ra docente de cada ciclo dentro del centro, las competencias van adquiriendo un significado diferente en cada caso. Esto nos debe hacer pensar que, cuando se formulan marcos generales prescriptivos e incluso marcos comunes de cualificación, se debe prever un importante margen de adaptación al contexto particular. Se trata más bien de orientaciones globales que deben tener un margen para la materialización local. Los equipos docentes y directivos desempeñan un papel fundamental en construir con sentido este margen entre el marco y la práctica educativa real.

\subsection{Caracteristicas de los procesos de enseñanza-aprendizaje}

Analizaremos la práctica de este caso de éxito haciendo un recorrido por su acercamiento metodológico, su concepción de la evaluación, los resultados de aprendizaje obtenidos y los nuevos roles.

\section{a) Metodología: aprendizaje basado en proyectos y retos}

Lo peculiar del caso tipo 2 es que las competencias transversales han dejado de ser la preocupación focal que ya aparece «expandida» a un marco más general de innovación metodológica de ciclo, en cuyo centro se encuentra el aprendizaje basado en retos. El profesorado ve la necesidad de modificar la metodología para poder enfrentarse a un proceso de aprendizaje coherente y acorde con el desarrollo efectivo de las CT, entendiendo siempre que eso es lo que las empresas del entorno demandan. Un reto no es un ejercicio (como ocurre en las prácticas tipo 1), sino que es una situación abierta que permite múltiples cierres y que mantiene a todas las partes implicadas en una situación de cierta incertidumbre. Así lo explica este profesor:

No es la clase magistral que la tienes preparada de pe a pa: sabes dónde empieza y dónde termina, o sea, tú sabes dónde vas a empezar y cómo vas a empezar. Un reto no sabes cómo lo vas a terminar y cuándo lo vas a terminar; un buen reto, claro. Un reto que lo diseñas en un momento, al final no es un reto, es un ejercicio, y no se trata de diseñar ejercicios, sino de diseñar retos que tengan distintas alternativas, distintas duraciones. Es decir, que un grupo pueda llegar a una solución, y otro grupo, a otra completamente distinta igual de buena, igual de fácil. (Profesor 2, caso tipo 2)

El reto como proyecto se vivencia como un entorno natural de aprendizaje para el desarrollo de las competencias transversales, en el que el alumnado va adquiriendo cada vez mayores niveles de autonomía. No es propiamente un acercamiento para desarrollar tal o cual CT de manera independiente, sino que el entramado metodológico crea una situación que funciona como nudo del desenvolvimiento de numerosos aprendizajes.

En los ciclos formativos de alto rendimiento, no hay una forma única de generar y desarrollar el reto. Cada ciclo tiene su «vida propia» al calor del contexto. Trabajar con retos supone perseguir objetivos comunes y superar diversas fases. Cada ciclo diseña qué fases va a tener el proyecto,cómo se van 
a trabajar (de forma individual o grupal), y el modo en la que se va a puntuar. No hay una guía que se pueda replicar en uno y otro lugar y funcione. Por el contrario, hay unas pautas comunes muy generales y algunas claves que se repiten en todos los casos estudiados. En concreto, las claves expresadas más recurrentes son:

- Visión de ciclo y de construcción de un lenguaje común.

- Desarrollo de retos reales con productos finales tangibles.

- Espacios de aprendizaje flexibles y capacidad de autogestionar el tiempo.

- Asegurar un compromiso por parte del alumnado.

En definitiva, estamos ante una situación en la que la atención se ha desplazado del desarrollo «fragmentado» de las CT, más característico de las tipologías 1 y 3 , al desarrollo de la capacidad de resolver retos como una capacidad aglutinadora del resto de competencias. El profesorado se da cuenta de que la clave se condensa en un cambio metodológico que crea las condiciones para que el alumnado desarrolle una actividad, el reto, que tiene un carácter profesional muy marcado y que le acerca al mundo laboral de forma constante.

\section{b) La evaluación de las competencias transversales}

La evaluación de las competencias transversales es uno de los aspectos que determina la diferencia entre los tres casos tipo en las buenas prácticas. En los casos tipo 1 y 2 , si bien no se sigue un procedimiento estandarizado ni tampoco se utiliza ningún instrumento específico, el profesorado reconoce, en su labor docente, la evaluación más o menos exhaustiva del trabajo en equipo, la autonomía, la capacidad de liderazgo, etc., sobre todo a partir de proyectos reales (en los casos individuales) o de retos (en los casos de ciclo). A diferencia de muchas de las prácticas de los tipos 1 y 3 , en los ciclos de alto rendimiento, tanto el alumnado como el profesorado señalan el seguimiento exhaustivo que se hace sobre todo de las actitudes y de las CT, así como el valor que adquieren en la calificación.

El seguimiento es uno de los escollos más grandes con los que se encuentra el profesorado dentro del módulo, la unidad y el reto. Como dirá un docente: «un talón de Aquiles». ¿Es necesario realizar un seguimiento exhaustivo de la competencia? ¿Significa hacer un seguimiento exhaustivo tener que estar observando y anotando continuamente en todas las unidades, horas lectivas, etc.? ¿Cómo hacer para ajustar el instrumento de medida al estilo docente de cada profesor o grupo de profesores? ¿Qué uso se puede realizar de las rúbricas? Son cuestiones que se plantean los docentes y que van resolviendo paulatinamente de forma más o menos particular.

A diferencia de aspectos actitudinales, como el uso de los EPI (Equipos de Protección Individual), la puntualidad o la asistencia, el seguimiento y la evaluación de las CT, por su carácter subjetivo y no tangible, resulta dificultoso tanto para el alumnado como para el profesorado, y las rúbricas hacen que, en ocasiones, el proceso resulte más complicado y pesado. 
El profesorado pone sobre la mesa una serie de interrogantes fundamentales que evidencian el tema de la calificación de las competencias transversales como un punto débil en la evaluación: ¿la calificación numérica recoge adecuadamente la competencia?, ¿hay que centrarse en el producto y/o en la evolución, el esfuerzo, etc.?, ¿`cuál es el modo más adecuado de certificar (registrar) coherentemente, de forma que sirva tanto al alumnado como al empleador? La siguiente voz ejemplifica bien la disyuntiva con la que se encuentra la mayoría del profesorado en su labor docente y evaluadora.

Hasta ahora las hemos puntuado en la evaluación final, o sea, les hemos dado un valor numérico, y esto es muy difícil. Yo calificar a un alumno en liderazgo con un 6 , con un 7 , con un 5 o con un $3 \ldots$ (Profesor 14, caso tipo 2)

Esta reflexión pone de manifiesto una crisis pedagógica interesante que supone una ruptura entre la concepción tradicional de la evaluación y una inmersión en planteamientos metodológicos innovadores, así como el cuestionamiento de la calificación numérica al uso, además de la certificación de la cualificación del alumnado en relación con estas competencias. La idea que va cogiendo fuerza es la del modelo de informe cualitativo como alternativa a la cartilla de notas tradicional.

Otros problemas que manifiesta el profesorado relacionado con la naturaleza más subjetiva e intangible de las competencias transversales son cómo recuperar una competencia transversal, una actitud, o cómo coordinar percepciones (valoraciones) divergentes entre módulos de profesorado sobre el mismo alumnado en relación con una CT. Este carácter menos tangible se hace más evidente en los contextos de coordinación del profesorado.

\section{c) Resultados de aprendizaje y valoración de la metodología}

En los ciclos de alto rendimiento, el alumnado tiene una conciencia más clara de las actitudes y de las competencias transversales tratadas que en las otras tipologías. Subrayan la labor relacionada con la autonomía, el desarrollo de las actividades en equipo y la comunicación, así como el efecto contagio y el valor motivacional que produce trabajar mediante retos.

Tal y como se aprecia en la siguiente voz, hay una percepción por parte del profesorado del efecto multiplicador que supone el trabajo con competencias transversales dentro del proceso formativo del alumnado.

Sí, este año lo he visto, lo he comprobado, y a decir verdad, sin esperarlo. No sabía nada sobre esto y todavía estoy aprendiendo, ¿no? Pero he comprobado que, integrando las transversales en los proyectos, al final, esto ha ayudado a ir adelante en el taller, ha ayudado a los alumnos y a las alumnas. Y esto no lo diría si no lo hubiera visto. No sé, me parece que se le ha añadido algo al trabajo del taller, la integración de las transversales, ¿̨no? (Profesor 16, T2)

También los hábitos de trabajo, las actitudes, adquieren, según el profesorado, un sentido diferente cuando están integrados en un contexto metodológico amplio e integrador, tal y como nos lo explica este docente: 
Ellos y ellas también deben adquirir el mismo compromiso, claro. Queremos dar otro paso con el asunto de la asistencia, pedir puntualidad y asistencia a todos, pero también funcionar como en una empresa. Si faltas, tendrás que recuperar. Hasta ahora, la política de la escuela es de un $15 \%$. No venir supone un $15 \%$, pero así se lo toman algunos y algunas. Lo suelen calcular justo, y lo que hemos hecho con esto es que esto no ocurra. (Profesor 10, T2)

Asimismo, algunas competencias transversales adquieren un sentido integrado particular en estos contextos de aprendizaje. El trabajo en grupo se concibe como una labor colaborativa "natural", y la competencia de aprender a aprender se asocia más con el desarrollo de la autonomía, señalada con expresiones como "buscarte la vida», "te ayudan a pensar por ti mismo", etc.

El alumnado constata y señala el efecto motivador del reto. Afrontar retos reales genera un aumento en la motivación y un efecto contagio para el aprendizaje y el trabajo. También el profesorado señala este valor, ya que posibilita activar competencias que difícilmente pueden ser desarrolladas en el modelo tradicional. Los logros alcanzados inciden exponencialmente en la motivación de los estudiantes, de forma que el grupo arrastra al estudiante individual.

Y ahí surge la dinámica. 20 alumnos y alumnas más 6 profesores y profesoras. Que uno se encuentra perdido es muy difícil. Lo que siempre comenta el alumnado respecto a esta metodología es que, si se le dejase solo, su expediente no sería el mismo, porque al final el grupo arrastra. Pero hay que dejar que el grupo arrastre. (Profesor 14, T2)

\section{d) Perfil profesional del profesor y la profesora. Nuevos roles del docente y del discente en la relación educativa}

La primera constatación es que los docentes entrevistados tienen muy claro para qué y por qué están haciendo lo que están haciendo. Son profesionales que operan con una intencionalidad educativa, más allá de la instructiva, muy claramente definida, que les lleva a implicarse considerablemente en la marcha $\mathrm{y}$ en el seguimiento del alumnado.

La intencionalidad pedagógica se hace patente en ideas como la necesidad de desarrollar las competencias transversales para obtener una cualificación profesional completa, asegurar el éxito académico del alumnado y motivarlo hacia el aprendizaje y la formación. En los ETHAZI, la implicación y la motivación del profesorado es clave para que se pueda llevar a cabo un reto a nivel de ciclo y un cambio metodológico similar. En esta línea, las prácticas de ciclo pueden ser interpretadas en el ámbito del profesorado reflexivo e investigador, en la medida en que generan un proceso dinámico y constante de discusión, selección y ajuste de las competencias y de las rúbricas correspondientes, muy cercanas procedimentalmente a la investigación-acción.

Otra de las cualidades que se señala como importante es la polivalencia, en el sentido de ser capaz de controlar varias materias, con lo que se rompe en cierta manera la fragmentación que trae consigo la especialización excesiva. 
Pero, para cualquier cosa, muchas veces también buscamos en el propio proyecto, pues, que un profesor pueda estar dando en un momento una materia, pero que también pueda dar otra. (Profesora 15, T2)

También consideran necesario mostrar un alto grado de resiliencia respecto al alumnado, lo que entraña, en definitiva, una actitud de expectativa alta respecto a su capacidad para resolver los retos que se le van a plantear, junto con un buen manejo de la frustración que puede derivarse de ello si no puede conseguirlo.

A fin de poder coordinarse y adaptarse, el profesorado considera clave la flexibilidad respecto a la materia impartida y a la metodología utilizada. En muchas ocasiones, ello supone que los docentes sean capaces de abandonar la zona pedagógica de confort a cambio de responder a lo que es prioritario para conseguir su objetivo: que el reto funcione lo mejor posible entre los estudiantes.

Y, aparte, a lo que has dicho tú, añadiría la flexibilidad. El propio proceso te exige ser flexible. Pues igual en una materia no tengo que profundizar tanto, tengo que pasarla más por encima porque se ajusta menos al proyecto. Eso también te exige cierta flexibilidad como profesor. Es importante no dar siempre de la misma manera ni lo mismo. Entonces eso también muchas veces es incómodo para un profesor. (Profesora 15, T2)

Si bien las características descritas son atribuibles también al profesorado de las otras tipologías, sin embargo, se condensan de forma más relevante en la de ciclo, es decir, cuando se ha producido ese proceso de autodeterminación del equipo docente en aras de desarrollar su proyecto pedagógico.

Por otra parte, la práctica de ETHAZI es la más ilustrativa y relevante para entender el papel que la coordinación desempeña en el desarrollo de las buenas prácticas. El profesorado subraya el carácter fundamental de la coordinación para planificar, poner en marcha y hacer el seguimiento del reto, para asegurar la integración en el grupo de docentes nuevos, así como para poder responder positivamente a los imprevistos que puedan surgir. No hay un modelo único de coordinación, sino que cada equipo pedagógico desarrolla su estilo propio y construye sus instrumentos para controlar la labor del profesorado y realizar el seguimiento del alumnado. Las claves del modelo de coordinación que subraya el profesorado de los equipos pedagógicos de ciclo entrevistados son: la sintonía entre el profesorado miembro del equipo docente, la integración tanto de los enseñantes nuevos como de módulos transversales (FOL, calidad, etc.), la igualdad en términos de estabilidad laboral entre los docentes y el liderazgo natural.

A su vez, el aprendizaje basado en proyectos y en retos exige un cambio en el rol de los docentes que no siempre es fácil de asumir. Su nuevo rol, dirán, supone dejar a un lado el patrón omnisciente y considerarse un profesional que aprende, que duda, que no tiene las respuestas para todo al momento, pero que sabe buscarlas y que, sobre todo, sabe acompañar el aprendizaje del alumnado. Es un tutor a quien le corresponde «mantenerse al margen», porque debe dejar espacio para que los estudiantes intenten hallar también sus res- 
puestas. Naturalidad y sinceridad se presentan como claves de la nueva relación educativa. La siguiente voz ilustra dicha percepción:

Mantenerles no, mantenerme al margen, en el sentido de decir: «Yo sé cuáles son los requisitos, pero no sé cuál es el camino para alcanzarlos». Eso es lo que te puedo decir. (Profesor 3, T3)

Como señala más de un profesor, una cualidad necesaria para un docente que quiera trabajar competencias entre los estudiantes será la de «predicar con el ejemplo». El reto con el que se enfrenta al alumnado es a la vez un reto pedagógico para el profesorado. Las competencias transversales que se pretenden desarrollar entre los estudiantes deben ser a la vez observadas en sus modelos, que no son otros que el equipo docente del ciclo. Las voces hablan muy elocuentemente de esta especie de isomorfismo.

\section{Discusión y conclusiones}

El impulso, a nivel europeo, de amplias políticas de formación basadas en competencias está teniendo un impacto perceptible en la formación profesional del País Vasco a nivel de profesorado, de alumnado y de equipos directivos, como ponen en evidencia los resultados de esta investigación. No obstante, las orientaciones prescriptivas que emanan de los diferentes niveles legales, normativos y profesionales se traducen de manera particular y contextual, $\mathrm{y}$, en este sentido, cabe corroborar que no hay una idea común que otorgue significado al concepto de competencia ni al de competencia transversal, tal y como se apunta en la literatura (Biemans et al., 2009; Mulder, 2007; Riesco, 2008). No hay unas competencias transversales universales y válidas para todos, sino lecturas contextualizadas y particularizadas para cada perfil profesional y para cada contexto docente (Weigel et al., 2007b).

El estudio de la FP vasca revela esta multiplicidad de «traducciones», de formas diferentes de superar los escollos apuntados por Biemans et al. (2009). Ello nos permite caracterizarlas como prácticas de éxito a nivel individual del docente que opta por desarrollarlas de forma intencional, a nivel de ciclo, en el que el equipo pedagógico se responsabiliza de manera colegiada en el desarrollo de los procesos de enseñanza-aprendizaje de forma global y a nivel institucional, en el que una política explícita ampara y orienta a la formación basada en competencias.

Si bien en los tipos 2 y 3, donde la decisión de trabajar las competencias transversales está más orquestada y acompañada por el equipo docente y/o por el equipo directivo, se alcanza un mayor nivel de sistematización y profundización conceptual, metodológica y relacional, así como una mayor conciencia y percepción del valor de las CT en el alumnado, el caso tipo 1 es el más extendido y, con mucha probabilidad, está presente en todos los centros, aunque es difícil de detectar de forma minuciosa. Es la semilla desde la que se impulsa el crecimiento y el tránsito hacia otras formas alternativas de curriculo en el ciclo. El tipo 3 desempeña un rol relacionado con la creación de 
condiciones favorables (materiales, organizativas, relacionales y de estatus) para el desarrollo de las CT.

El análisis detallado de los tres casos tipo presentados revela que el nivel de ciclo (tipo 2) y, en particular, la experiencia de los ciclos de alto rendimiento, al menos en la FP vasca, es el que presenta mayor relevancia y significación desde el punto de vista del desarrollo de la competencias transversales. En estos casos, el ciclo se convierte en un hábitat favorable para hacer eclosionar procesos de cambio metodológico que incorporen el trabajo en competencias de manera integrada y global. La atención no se centra tanto en desarrollar tal o cual competencia, sino que el hecho de desarrollarlas sea el producto de plantear un reto o un proyecto que aglutine un proceso complejo de enseñanzaaprendizaje (Perrenoud, 2012). Los resultados en términos de aprendizaje han sido relevantes. Aun así, consideramos que los niveles de satisfacción recogidos en nuestro estudio deberían de ser completados en un futuro con un análisis más exhaustivo de los grados de consecución de competencias, sobre todo desde la perspectiva de los empleadores y/o de las empresas que colaboran con los centros.

Atendiendo a los 8 principios del modelo de desarrollo presentado por Wesselink, Biemans, Mulder y Van den Elsen, 2007 (Wesselink, 2010; Sturing, Biemans, Mulder y De Bruijn, 2010; Mulder, 2012), podemos decir que, en líneas generales, representan lo que se ha propuesto como el paso de una «educación orientada a las competencias» a un "modelo comprensivo de educación y formación basado en competencias». Si bien las claves que caracterizan a la tipología de ciclo son más relevantes que los casos correspondientes a los tipos 1 y 3 en aspectos relacionados con la definición de competencias, el diseño del currículo en referencia a problemas profesionales, la relación entre aprendizaje y vida real, el rol del profesorado como formador y experto, la actitud hacia el aprendizaje y la identidad profesional. Sin embargo otros aspectos, como la evaluación y la autorresponsabilidad del alumnado, necesitan todavía un mayor desarrollo.

Los ETHAZI son el ejemplo que concentra esta innovación, pero su génesis y su desarrollo requieren de condiciones especiales que no siempre se encuentran de forma natural en la realidad de los centros. El hecho de que el equipo docente implicado deje a un lado la visión basada en las materias y sus programaciones, y diseñe, desarrolle y evalúe retos es un cambio pedagógico de gran envergadura cultural, organizativa e incluso política. Es evidente que no es posible realizar dicho cambio sin que esos docentes lo hayan experimentado previamente a nivel individual y hayan llegado a la conclusión de que no se puede realizar una formación basada en competencias sin poner en crisis determinadas formas curriculares y didácticas muy arraigadas en nuestro sistema docente. Tampoco se pueden establecer formas curriculares alternativas, como la de la tipología de ciclo, sin una colaboración ni un apoyo activo de las direcciones de los centros e incluso de todo el entramado institucional (Escudero, 2012). 


\section{Referencias bibliográficas}

Arguelles, A. y Gonczi, A. (2000). Competency Based Education and Training: A world perspective. México: Conalep/Noriega.

Arias-Gundin, O.; Fidalgo, R. y García, J.N. (2008). El desarrollo de las competencias transversales en magisterio mediante el aprendizaje basado en problemas y el método de caso. Revista de Investigación Educativa, 26(2), 431-444.

Benito, A. y Cruz, A. (2005). Nuevas claves para la docencia universitaria en el EEES. Madrid: Narcea.

Biemans, H.; Wesselink, R.; Gulikers, J.; Schaafsma, S.; Verstegen, J. y Mulder, M. (2009). Towars competence-based VET: Dealing with pitfalls. Journal of Vocational Education and Training, 61(3), 267-286.

Bisquerra, R. (coord.) (2004). Metodología de la investigación educativa. Madrid: La Muralla.

Blanco, A. (2008). La rúbrica útil para la evaluación de competencias. En L. Prieto (coord.). La enseñanza universitaria centrada en el aprendizaje (pp. 171-188). Barcelona: Octaedro.

Bogdan, R.C. y BiKLEN, S.K. (1982). Qualitative research for education: An introduction to theory and methods. Boston: Allyn and Bacon.

Bolívar, A. (2008). El discurso de las competencias en España: Educación básica y educación superior. Red U. Revista de Docencia Universitaria, número monográfico 2, 6(2), 1-23.

Bridge, S.; O’Neill, K. y Cromie, S. (2003). Understanding Enterprise, Entrepreneurship and Small Business. Basingstoke: Palgrave.

CONFEBASK (2010). Una decidida apuesta por la formación. <http://issuu.com/ confebask/docs/revista-confebask-2010-n1/1>

Descy, P. y Tessaring, M. (2001). Training and learning for competence. Second report on vocational training research in Europe: Synthesis report. Luxemburgo: Cedefop. Recuperado de cedefop.europa.eu

EsCUDERO, J.M. (2009). Buenas prácticas y programas extraordinarios de atención al alumnado en riesgo de exclusión educativa. Profesorado: Revista de Curriculum y Formación del Profesorado, 13(3), 107-141.

- (2012). La colaboración docente, una manera de aprender juntos sobre el trabajo cooperativo con el alumnado. En A. Torrego y A. Negro (coord.). Aprendizaje cooperativo en las aulas (pp. 269-289). Madrid: Alianza Editorial.

European Commission/EACEA/Eurydice (2012). Developing Key Competences at School in Europe: Challenges and Opportunities for Policy. Luxemburgo: Publications Office of the European Union.

Eusko Jaurlaritza / Gobierno Vasco. IV Plan Vasco de Formación Profesional: Hacia una FP diferente. <https://www.irekia.euskadi.eus/uploads/attachments/5674/ IV_Plan_vasco_de_formacion_profesional_18-12-2014_DEF.pdf>

Fernández March, A. (2006). Metodologías activas para la formación de competencias. Educatio Siglo XXI, 24, 35-56.

Gimeno Sacristán, J. (coord.) (2008). Educar por competencias. ¿Qué hay de nuevo? Madrid: Morata.

Gómez, J.; Latorre, A.; Sánchez, M. y Flecha, R. (2007). Metodología comunicativa critica. Barcelona: El Roure.

Grootings, P. (1994). De la cualificación a la competencia: ¿De qué se habla? Revista Europea de Formación Profesional, 1, 5-8. 
Guba, E.G. y Lincoln, Y.S. (1985). Naturalistic Inquiry. Londres: Sage.

Gulikers, H. (2006). Authenticity is in the eye of the beholder. Netherlands Open University. Disertación doctoral no publicada.

Hargreaves, A. (2003). Enseñar en la sociedad del conocimiento. Barcelona: Octaedro. HETEL (2008). Competencias clave en la Formación Profesional: Aportaciones sobre cómo trabajarlas y cómo evaluarlas. <https://issuu.com/hetel/docs/informe_ completo_con_indice_compete>

Imbernon, F. y Medina, J.L. (2006). Metodología participativa en el aula universitaria: La participación del alumnado. En M. Martínez y S. Carrasco. Propuestas para el cambio docente en la universidad (pp. 91-122). Barcelona: Octaedro.

INCUAL (2002). Consejos y programas de formación profesional. Madrid: INEM. Colección Informes.

Knight, P. y Yorke, M. (2003). Employability and Good Learning in Higher Education. Teaching in Higher Education, 8(1), 3-16.

Krueger, R.A. (1991). El grupo de discusión: Guia práctica para la investigación aplicada. Madrid: Ediciones Pirámide.

Miguel, M. de (dir.) (2005). Modalidades de enseñanza centradas en el desarrollo de competencias: Orientaciones para promover el cambio metodológico en el espacio europeo de educación superior. Oviedo: Universidad de Oviedo.

Monereo, C. (coord.) (2009). PISA como excusa: Repensar la evaluación para cambiar la enseñanza. Barcelona: Graó.

Moya, J. (2008). Las competencias básicas en el diseño y el desarrollo del currículo. Qurriculum, 21, 57-78.

Mulder, M. (2007). Competencia: La esencia y la utilización del concepto en la formación profesional inicial y permanente. Revista Europea de Formación Profesional, 40(1), 5-24.

- (2012). Developing comprehensive competence-based education and training: Some practical guidelines. Journal of Agricultural Education and Extension, 18(3), 305-314.

OCDE (2005). La definición y selección de competencias clave (DeSeCo). Resumen ejecutivo. Traducción en versión electrónica, 1-20. <http://deseco.ch/bfs/deseco/en/ index/03/02.parsys.78532.downloadList.94248.DownloadFile.tmp/2005.dscexecutivesummary.sp.pdf> [29 de enero de 2016]

Отт, В. (1999). Características estructurales y categorías de destinatarios para una formación profesional integral. Revista Europea de Formación Profesional, 17, 57-67.

Perrenoud, P. (2004). Diez nuevas competencias para enseñar. Barcelona: Graó.

- (2012). Cuando la escuela pretende preparar para la vida: ¿Desarrollar competencias $o$ enseñar otros saberes? Barcelona: Graó.

Poblete, M.; Bezanilla, M.J.; Fernández-Nogueira, D. y Campo, L. (2016). La formación del docente en competencias genéricas: Un instrumento para su planificación y desarrollo, Educar, 52(1), 71-91. <https://doi.org/10.5565/rev/educar.713>

Prahalad, C.K. y Hamel, G. (1990). The core competence of the corporation. Harvard: Business Review.

Proyecto Tuning (2003): Tuning Educational Structures in Europe. Informe final. Proyecto piloto. Fase 1. Bilbao: Universidad de Deusto.

Rauner, F. (2007). El conocimiento práctico y la competencia de acción en la profesión. Revista Europea de Formación Profesional, 40(1), 58-73. 
Riesco, M. (2008). El enfoque por competencias en el EESS y sus implicaciones en la enseñanza y el aprendizaje. Tendencias Pedagógicas, 13, 79-105.

Rodríguez, A. (2012). Orientación profesional por competencias transversales para mejorar la empleabilidad. Universidad de Zaragoza. Ciencias de la Educación. Repositorio de la Universidad de Zaragoza. Tesis doctoral. <https://zaguan.unizar.es/ record/9591? $\ln =$ es $>$

Rodríguez Gómez, G.; Gil Flores, J. y García Jiménez, E. (1996). Metodología de la investigación cualitativa. Málaga: Aljibe.

RuÉ, J. (2009). El aprendizaje autónomo en educación superior. Madrid. Narcea.

Sánchez Gómez, M.C.; García-Valcárcel Muñoz-Repiso, A. (2001). La función docente del profesorado universitario. Bordón, 53(4), 581-595.

STAKe, R.E. (1998). Investigación con estudio de casos. Madrid: Morata.

Sturing, L.; Biemans, H.J.A.; Mulder, M. y Bruijn, E. de (2010). To What Extent are Vocational Courses Competence-Based?: Evaluation of a Model for Self-Assessing Competence-Based Vocational Education. Comunicación presentada en la European Conference on Educational Research, 1-12. Helsinki.

Vázquez Recio, R. y Angulo Rasco, F. (2003). Introducción a los estudios de casos: Los primeros contactos con la investigación etnográfica. Archidona (Málaga): Aljibe.

Villardón-Gallego, L. (coord.) (2015). Competencias genéricas en educación superior: Metodologías especificas para su desarrollo. Madrid: Narcea.

Wageningen UR. Education and Competence Studies Group. <http://www.wur.nl/en/ Expertise-Services/Chair-groups/Social-Sciences/Education-and-CompetenceStudies-Group.htm>.

Weigel, T.; Mulder, M. y Collins, K. (2007a). The concept of competence in the development of vocational education and training in selected EU member states. Journal of Vocational Education and Training, 59(1), 53-66.

- (2007b). The concept of competence in the development of vocational education and training in selected EU member states: A critical analysis. Journal of Vocational Education and Training, 59(1), 67-88.

WESSELINK, R. (2010). Comprehensive competence-based vocational education: The development and use of a curriculum analysis and improvement mode. Wageningen: Wageningen University and Research Center.

Wesselink, R.; Biemans, H.J.A.; Mulder, A. y Van den Elsen, E. (2007). La formación profesional basada en competencias vista por los investigadores neerlandeses. Revista Europea de Formación Profesional, 40(1), $42-57$.

YIN, R.K. (2003). Case Study Research: Design and Methods. 3. ${ }^{\mathrm{a}}$ ed. Londres: SAGE. 